\title{
Comparison Chosen DFT Methods with Six Basis Sets on Harmine Molecule
}

\author{
Maryam Malekzadeh* and Emran Heshmati \\ Department of biology, Parand Branch, Islamic Azad University, Tehran, Iran. \\ ${ }^{*}$ E-mail: m.malekzade62@gmail.com
}

(Received July 25, 2012; Accepted November 23, 2012)

\begin{abstract}
The effect of the DFT methods and basis sets on harmine molecule has been investigated. 26 DFT methods with 6 basis sets were used. Two main comparison chosen DFT methods in this work has been investigated. It is concluded that this contribution is very important and cannot be neglected. In the following analysis, changes in energy levels were investigated by two different methods. Considering a specific basis set, changes in energy levels were obtained using different DFT methods. A specific DFT method is chosen and changes in energy levels have been investigated by means of various basis sets. Effect of the choice of basis sets on geometrical parameters on harmine molecule has also been investigated.
\end{abstract}

Key words: DFT method, Harmine molecule, Basis sets, Geometrical parameters

\section{INTRODUCTION}

Density functional theory (DFT) is a quantum mechanical modeling method used in physics and all fields of chemistry to investigate the electronic structure (principally the ground state) of many-body systems, in particular atoms, molecules, and the condensed phases. ${ }^{1}$ With this theory, the properties of a many-electron system can be determined by using functional, i.e. functions of another function, which in this case are the spatially dependent electron densities. Hence the name density functional theory comes from the use of functional of the electron density. DFT is among the most popular and versatile methods available in condensed-matter physics, computational physics, and computational chemistry. DFT has been very popular for calculations in solid-state physics since the 1970s. However, DFT was not considered accurate enough for calculations in quantum chemistry until the 1990s, when the approximations used in the theory were greatly refined to improve the exchange and correlation interactions modeling. In many cases the results of DFT calculations for solidstate systems agree quite satisfactorily with experimental data. Computational costs are relatively low when compared to traditional methods, such as Hartree-Fock theory and its descendants based on the complex many-electron wave function. Since there are a large variety of DFT methods, choosing appropriate method and basis set are of crucial importance especially when dealing with large biological molecules with hetero-aromatic rings. Previous studies show that select and use of appropriate methods and com- putational tasks and basis sets are related to the structure under investigation. ${ }^{12,23}$ The aim of this work is the comparison of a series of different DFT methods and basis sets, in order to find a more suitable method and basis set for similar investigation. For this purpose we chose a medium size molecule, harmine (Fig. 1), (7-Methoxy-1methyl-9H-pyrido [3,4-b] indole) that is comparable with biological monomers such as nucleotides and amino acids. ${ }^{4}$ Harmine originally isolated from the seeds of Peganum harmala, is a tricyclic compound belonging to the $\beta$-carboline alkaloids. $\beta$-carboline such as harmine and harmaline constitute over than $60 \%$ of the alkaloids present in the plant seeds.

These alkaloids possess a broad range of pharmacological activities, such as anxiolytic and behavioral effects. ${ }^{5,6}$ Some studies demonstrated that harmine possessed significant anti-tumor potential both in vitro and in vivo. ${ }^{7-9}$ Anti-bactericidal $^{10}$ and anti-inflammation. ${ }^{11,12}$ Also harmine

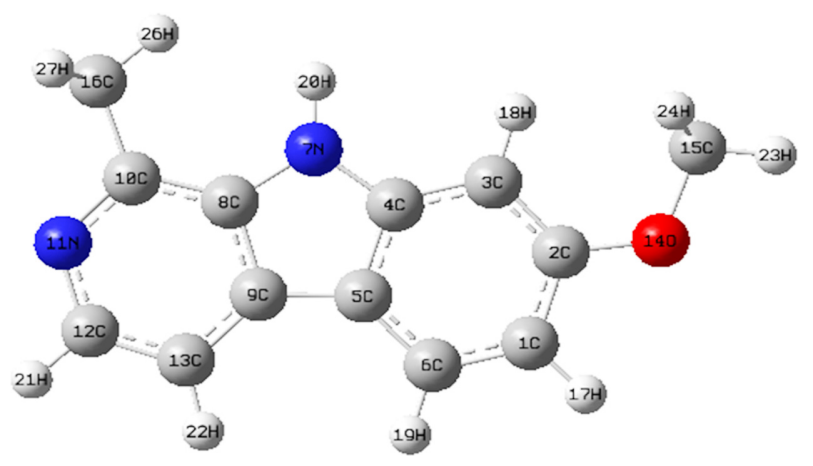

Fig. 1. Scheme of structure of the Harmine molecule. 
has effects on central nervous system, ${ }^{13,14}$ bone system ${ }^{15}$ and enzyme systems. ${ }^{16,17}$

\section{METHOD AND THEORY}

Gaussian 03 program was used for all quantum chemical computations performed in this study. ${ }^{18}$ Major computations were carried out using different DFT methods in combination with different basis sets. Also a MP2 computations under $6-31+\mathrm{G}^{*}$ and $6-31 \mathrm{G}^{*}$ basis sets was carried out for comparison. For all computations the starting geometry of harmine was the same and all computations were carried out in gas phase.

A brief introduction about the different DFT methods is given in the Table 1. More comprehensive details can be

Table 1. Optimized harmine energy (J) obtained from MP2 computations

\begin{tabular}{ll}
\hline $\mathrm{MP} 2 / 6-31 \mathrm{G}^{*}$ & $2.977870684 \times 10^{-15}$ \\
\hline $\mathrm{MP} 2 / 6-31+\mathrm{G}^{*}$ & $2.977936554 \times 10^{-15}$ \\
\hline
\end{tabular}

found in. ${ }^{19-23}$ According to Table 1 the DFT methods used in this study can be divided into four groups on the basis of their exchange functional. They are hybrid, ${ }^{24}$ Beckes 88 exchange functional, ${ }^{25}$ the Xa exchange functional, ${ }^{26}$ and Slaters exchange functional, ${ }^{27}$ based DFT methods. These four groups have a few different DFT methods that are derived from the three correlation functional of Perdew 86 (P86), ${ }^{28}$ Perdew-Wang 91(P91), ${ }^{29}$ and Lee-Yang-Parr(LYP) ${ }^{30}$ They are also combined with Perdew Local (PL), ${ }^{30}$ and Vosko-Wilk-Nusair (VWN) ${ }^{31}$ correlation functional. Since the purpose of this calculation on harmine molecule is to compare DFT methods with different basis sets, 26 DFT methods that mentioned in Table 1 were chosen along with 6 different basis sets to calculate the exact level of optimized energy. We used different basis sets for all the atoms. A final refinement of the basis set implies the introduction of diffuse functions ${ }^{32}$ for heavy atoms which are supplemented with diffuse s- and p-type functions which leads to $6-31+\mathrm{G}^{*}$ and $6-31 \mathrm{G}^{* *}$ sets. A higher degree of splitting is possible with the $6-311 \mathrm{G}$ basis set producing

Table 2. Full list of the DFT methods available in Gaussian 03

\begin{tabular}{|c|c|c|}
\hline Functional & Gaussian 03 key word & Describe \\
\hline$\overline{\mathrm{B} 3 \mathrm{LYP}}$ & B3LYP & Beckes three parameter functional in with combination LYP correlation functional \\
\hline BLYP & BLYP & Beckes functional in with combination LYP correlation functional \\
\hline BVWN & BVWN & Beckes functional in with combination VWN correlation functional \\
\hline BPW91 & BPW91 & Beckes functional in with combination PW91correlation functional \\
\hline B3PW91 & B3PW91 & Beckes three parameter functional in with combination PW91 correlation functional \\
\hline B3P86 & B3P86 & Beckes three parameter functional in with combination P86 correlation functional \\
\hline BP86 & BP86 & Beckes functional in with combination LYP correlation functional \\
\hline PW91VWN & PW91VWN & Gradient-corrected correlation functional in with combination VWN correlation functional \\
\hline PW91PL & PW91PL & Gradient-corrected correlation functional in with combination PL correlation functional \\
\hline PW91P86 & PW91P86 & Gradient-corrected correlation functional in with combination P86 correlation functional \\
\hline PW91LYP & PW91LYP & Gradient-corrected correlation functional in with combination LYP correlation functional \\
\hline G96LYP & G96LYP & G96 hybrid functional in with combination LYP correlation functional \\
\hline G96P86 & G96P86 & G96 hybrid functional in with combination P86 correlation functional \\
\hline G96VWN & G96VWN & G96 hybrid functional in with combination VWN correlation functional \\
\hline G96PL & G96PL & G96 hybrid functional in with combination PL correlation functional \\
\hline MPWLYP & MPWLYP & Barone modified PW91 in with combination LYP correlation functional \\
\hline MPWP86 & MPWP86 & Barone modified PW91 in with combination P86 correlation functional \\
\hline XaVWN & XaVWN & Xa exchange functional in with combination VWN correlation functional \\
\hline $\mathrm{XaPL}$ & $\mathrm{XaPL}$ & Xa exchange functional in with combination PL correlation functional \\
\hline XaLYP & XaLYP & Xa exchange functional in with combination LYP correlation functional \\
\hline MPWVWN & MPWVWN & Barone modified PW91 in with combination VWN correlation functional \\
\hline MPWPL & MPWPL & Barone modified PW91 in with combination PL correlation functional \\
\hline SLYP & SLYP & Slaters exchange functional in with combination LYP correlation functional \\
\hline SVWN & SVWN & Slaters exchange functional in with combination VWN correlation functional \\
\hline SVWN5 & SVWN5 & Slaters exchange functional in with combination the fifth correlation functional of VWN \\
\hline SP86 & SP86 & Slaters exchange functional in with combination P86 correlation functional \\
\hline SPL & SPL & Slaters exchange functional in with combination PL correlation functional \\
\hline
\end{tabular}


Table 3. Optimized Harmine energy ( $\mathrm{J}$ ) obtained from DFT methods and basis sets

\begin{tabular}{|c|c|c|c|c|c|c|}
\hline Methods & $6-31 G^{*}$ & $6-31 \mathrm{G}^{*}$ & $6-31 G^{* *}$ & $6-311 \mathrm{G}$ & $6-311 G^{*}$ & $6-311 G^{* *}$ \\
\hline $\mathrm{HF}$ & -2.976703678 & -2.977896909 & -2.977995884 & -2.977277488 & -2.97845141 & -2.97854522 \\
\hline BLYP & -2.994744257 & -2.99548295 & -2.995566637 & -2.995474774 & -2.996179569 & -2.996266169 \\
\hline B3LYP & -2.995852797 & -2.997284087 & -2.996755163 & -2.996516241 & -2.997310688 & -2.997398358 \\
\hline BVWN & -3.019409539 & -3.020137639 & -3.020221988 & -3.020112253 & -3.020815362 & -2.997047559 \\
\hline BPW91 & -2.995606746 & -2.996338702 & -2.996418078 & -2.996249786 & -2.996959505 & -2.997047559 \\
\hline B3PW91 & -2.994737811 & -2.995548982 & -2.995630408 & -2.995345065 & -2.99613937 & -2.996227861 \\
\hline В3P86 & -3.004668618 & -3.005483536 & -3.005565611 & -3.005289325 & -3.006087978 & -3.006175868 \\
\hline BP86 & -2.995935318 & -2.996666766 & -2.996746337 & -2.996600031 & -2.997309564 & -2.997396146 \\
\hline PW91VWN & -3.01863968 & -3.019374642 & -3.019460137 & -3.019361813 & -3.020069348 & -3.020157441 \\
\hline PW91PL & -3.008965893 & -3.009696572 & -3.00978154 & -3.009690268 & -3.010395521 & -3.010481007 \\
\hline PW91P86 & -2.995167912 & -2.995906005 & -2.995986768 & -2.995850419 & -2.996564398 & -2.996650506 \\
\hline PW91LYP & -2.993977761 & -2.994723898 & -2.994808738 & -2.994730885 & -2.995439971 & -2.995526088 \\
\hline G96LYP & -2.994530691 & -2.995267494 & -2.995348222 & -2.995245888 & -2.995947751 & -2.996034156 \\
\hline G96P86 & -2.995726287 & -2.996456118 & -2.996532813 & -2.99638014 & -2.997086853 & -2.997173245 \\
\hline G96VWN & -3.019197668 & -3.019923968 & -3.02000544 & -3.011988773 & -3.020587703 & -3.020676391 \\
\hline G96PL & -3.009523419 & -3.010245408 & -3.01032636 & -3.010214743 & -3.01091378 & -3.010998478 \\
\hline MPWLPY & -2.994717687 & -2.995459208 & -2.995543403 & -2.995461344 & -2.996167853 & -2.996254212 \\
\hline MPWP86 & -2.995907749 & -2.996641602 & -2.996721701 & -2.996582961 & -2.997294308 & -2.99738065 \\
\hline MPWPL & -3.009708383 & -3.010434793 & -3.010519122 & -3.01042469 & -3.011125239 & -3.011213096 \\
\hline MPWVWN & -3.019382013 & -3.02011272 & -3.020197571 & -3.020096244 & -3.020801148 & -3.020889554 \\
\hline XaLYP & -2.957529861 & -2.973029046 & -2.973890007 & -2.97383787 & -2.974592747 & -2.974679636 \\
\hline $\mathrm{XaPL}$ & -2.987971609 & -2.988809867 & -2.988885914 & -2.988739792 & -2.989488018 & -2.989576121 \\
\hline XaVWN & -2.997656314 & -2.998498521 & -2.998575011 & -2.998422826 & -2.999175181 & -2.999263842 \\
\hline SLYP & -2.954680147 & -2.95557084 & -2.955644194 & -2.955514899 & -2.956248861 & -2.95334099 \\
\hline SVWN & -2.979279246 & -2.980148042 & -2.980221447 & -2.98006493 & -2.9807964 & -2.980883335 \\
\hline SVWN5 & -2.96977952 & -2.970640411 & -2.970719905 & -2.970568033 & -2.971297183 & -2.971383736 \\
\hline
\end{tabular}

the resulting $6-311 \mathrm{G}^{*}$ and $6-311 \mathrm{G}^{* *}$ sets.

\section{RESULTS AND DISCUSSION}

The purpose of this calculation on harmine molecule is to compare DFT methods with different basis sets. For this purpose, 26 DFT methods mentioned in Table 1 were chosen along in combination with 6 different basis sets to calculate the exact level of optimized energy. The results are shown in Table 3. According to these results, the minimum energy is related to SLYP followed by SP86, SPL, SVWN5 and XaLYP respectively. On the other hand, the maximum energy is related to BVWN followed by MPWVWN, G96VWN, PW91VWN and G96PL, respectively. Chosen DFT methods listed in Table 2 were investigated in two different manners which yield two sets of results. DFT methods that show linear increase in rate of energy with a specific basis set are G96P86, G96LYP, PW91P86, PW91LYP and MPWLYP, which are illustrated in plot 2. On the other hand, there are DFT methods that was investigated with each basis set one by one. Based on plots 3 to 8 similar results were obtained for each method. In this manner the minimum energy level was related to basis set 6-31G, and the maximum energy level was related with higher degree of splitting such as 6-311 $\mathrm{G}^{*}$ and 6-311 $\mathrm{G}^{* *}$. Different behavior of basis set $6-31 \mathrm{G}^{*}$ is noticeable in these plots.

\section{Effect of the Basis Sets on Geometrical Parameters}

Bond angels: Bond angels of harmine molecule were investigated using 26 DFT methods and basis sets. Proposed angels were $10 \mathrm{C}-11 \mathrm{~N}-12 \mathrm{C}$ and $8 \mathrm{C}-7 \mathrm{~N}-4 \mathrm{C}$ (Fig. 1). The bond angels show small dependency in the choice of the basis sets for smaller ring. Results for $\mathrm{C}-\mathrm{N}-\mathrm{C}$ bond angels are illustrated in Figures 9-34(result are presented in supplementary data file). It shows very small discrepancy between all the basis sets. Results obtained for 6-31G*, 6$31 \mathrm{G}^{* *}, 6-311 \mathrm{G}^{*}$ and $6-311 \mathrm{G}^{* *}$ are very similar and the results for 6-31G and 6-311G are exactly equivalent.

Bond length: Bond lengths of harmine molecule are investigated using 26 DFT methods and 6 basis sets. The 


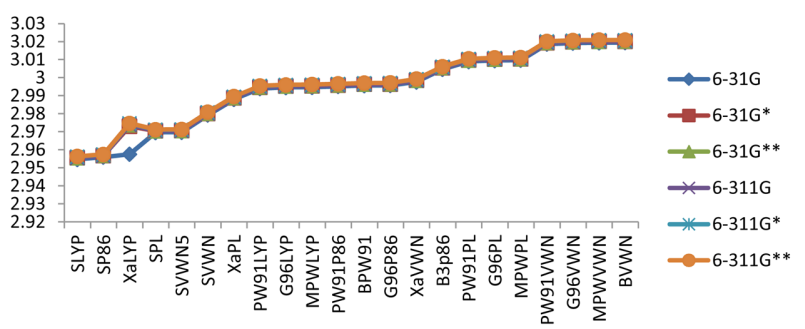

Fig. 2. harmine optimized energy (J) obtained from DFT methods with six basis sets.

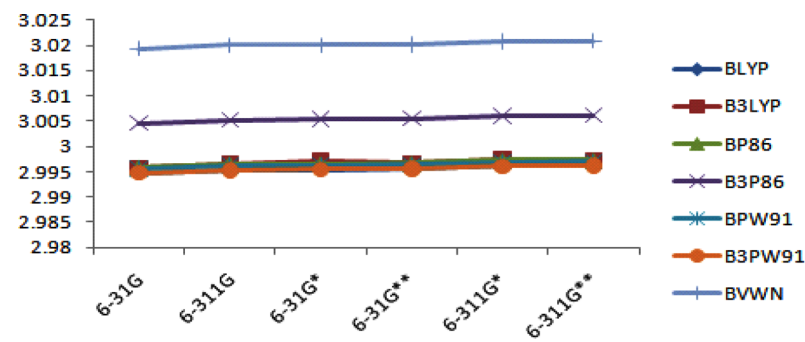

Fig. 3. harmine molecule optimized energy using by Beckes combination functional on basis sets.

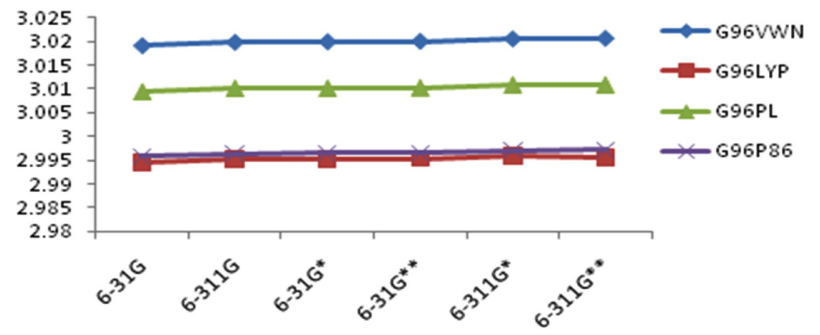

Fig. 4. harmine molecule optimized energy using by G96 combination functional on basis sets.

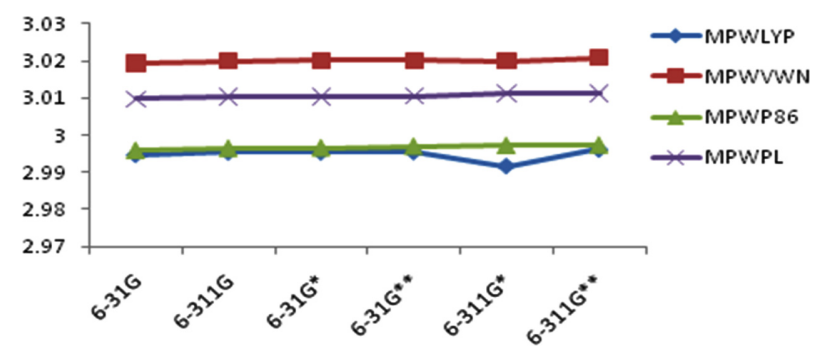

Fig. 5. harmine molecule optimized energy using by MPW combination functional on basis sets.

bond length of $11 \mathrm{~N}-12 \mathrm{C}, 10 \mathrm{C}-11 \mathrm{~N}, 7 \mathrm{~N}-4 \mathrm{C}$ and $8 \mathrm{C}-7 \mathrm{~N}$ for basis sets comparison is proposed (Fig. 1). Results obtained for all 28 DFT methods are very similar, but the results showed a very different behavior for PW91PL, PW91P86, PW91VWN, PW91LYP, XaPL and XaVWN. The minimal basis sets (6-31G and 6-311G) showed a maximum length of $\mathrm{C}-\mathrm{N}$ bonds for both rings. Figures 35 to 63 (result are presented in supplementary data file).

MP2 Calculation: The optimized energy of harmine

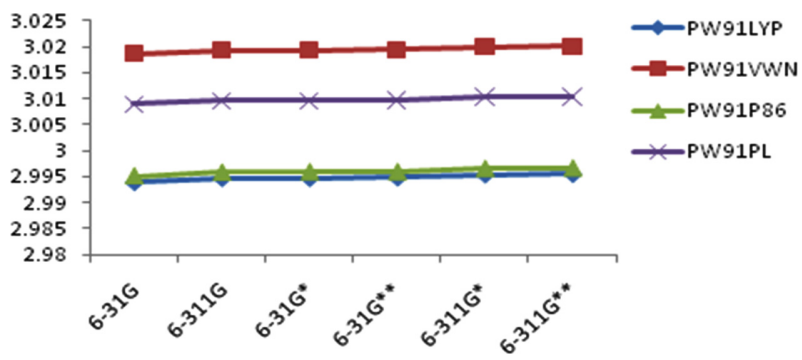

Fig. 6. harmine molecule optimized energy using by PW91 combination functional on basis sets.

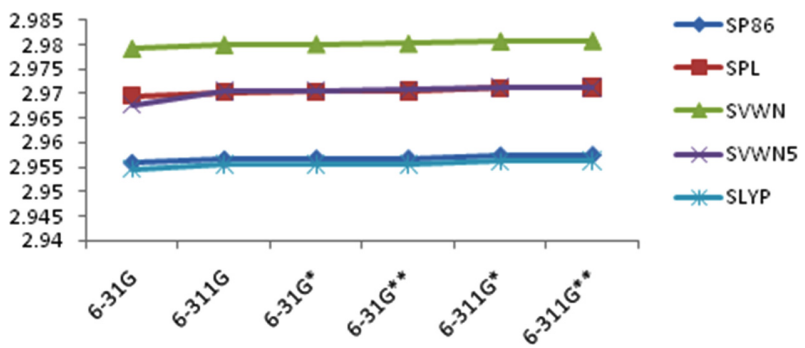

Fig. 7. harmine molecule optimized energy using by $\mathrm{S}$ exchange functional on basis sets.

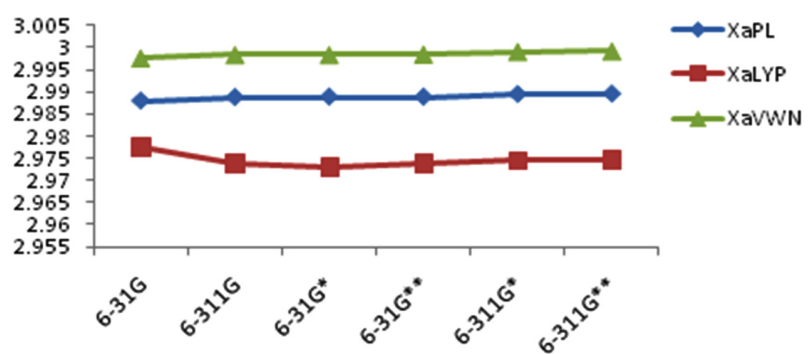

Fig. 8. harmine molecule optimized energy using by Xa exchange functional on basis sets.

molecule was calculated using MP2 method with 6-31G* and $6-31+\mathrm{G}^{*}$ basis sets. Results are shown in Table 2 .

Our MP2 results show that there is no significant difference between MP2 and difference DFT energies. In other words; different DFT methods have the same accuracy as MP2 in energy calculations. So that DFT methods are preferred, because of some other advantages such as computation time.

\section{CONCLUSION}

Optimum energy level of harmine molecule is compared using 26 DFT methods and 6 basis sets (Table 2). Results estimate that the minimum energy occurred while using Slater functional (SLYP, SP86 and SPL, Respectively) and the maximum energy occurred while using combination functional (PW91VWN, G96VWN, BVWN 
and MPWVWN, respectively). Tables 1 and 2 and figures show the different rate of energy level changes for LYP, P86, PL and VWN, with exchange or combination functional. It is concluded that the increase in exchange functional energy level varies with correlation functional and it yields same results for every exchange functional. The maximum energy level was obtained from exchange functional with VWN and PL, P86 correlation functional, respectively. On the other hand, the minimum energy level is obtained with LYP correlation functional. It appears from these results that for compression chosen DFT methods in this work harmine molecule do not sufficient. For more accurate results in investigation of methods with different basis sets, we recommend using bigger bio molecules specially proteins and nucleotides acids.

\section{REFERENCES}

1. Capelle, K. A Bird's-Eye View of Density-Functional Theory. Braz. J. Phy. 2006, 36(4A).

2. Burke, K. Perspective on Density Functional Theory. $J$. Chem. Phy. 2012, 136, 150901.

3. Nedelec, J. M.; Hench, L. L. Effect of Basis Set and of Electronic Correlation on Silica Rings. J. Non-Cryst. Solids 2000, 277, 106-113.

4. Murray, T. D.; Berger, A. Alcohol Withdrawal. Va. Med. Q 1997, 124, 184-187.

5. Brierley, D. I.; Davidson, C. Developments in Harmine Pharmacology Implications for Ayahuasca Use and Drug-dependence Treatment. Progress in Neuro-Psychopharmacology \& Biological Psychiatry 2012, 39, 263-272.

6. Almbladh, C. O.; Barth, U. V. Phys. Rev. 1985, 1331, 3231.

7. Perez Martin, J. M.; Labrador, V.; Fernandez Freire, P.; Molero, M. L.; Hazen, M. J. Ultrastructural Changes Induced in HeLa Cells After Phototoxic Treatment with Harmine. J. Appl. Toxicol. 2004, 24, 197-201.

8. Levy, M. Phys. Rev. 1982, A26, 1200.

9. Kohnand, W.; Sham, L. J. Phys. Rev. 1965, 140, A1133.

10. Ashad, N.; Zitterl-Eqlseer, K.; Hasnain, S.; Hess, M. Effect of Peganum Harmala or Its Beta-carboline Alkaloids on Certain Antibiotic Resistant Strains of Bacteria and Protozoa from Poultry. Phytother. Res. 2008, 22(11), 1533-1538.

11. Shahverdi, A. R.; Monsef-Esfahani, H. R.; Nickavar, B.; Bitarafan, L.; Khodaee, S.; Khoshakhlagh, N. Antimicrobial Activity and Main Chemical Composition of two Smoke Condensates from Peganum Harmala Seeds. Z. Naturforsch., C: J. Biosci. 2005, 60(9-10), 707.

12. Fortunato, J. J.; et al. A Cute Harmine Administration Induces Antidepressive-like Effects and Increases BDNF Levels in the Rat Hippocampus. Prog. Neuropsychopharmacol Biol. Psychiatry 2009, 33(8), 1425-1430.

13. Farzin, D.; Salimi, E. Effects of Harmane, Norharmane and Harmine on Apomorphine-induced Pecking Behavior in Chick. Mazandaran Uni. Med. Sciences 2009, 19(70), 747.

14. Yonezawa, T.; et al. Harmine, $\alpha \beta$-carboline Alkaloid, Inhibits Osteoclast Differentiation and Bone Resorption in Vitro and in Vivo. Eur J. Pharmacol. 2011, 650(2-3), 511-518.

15. Adayev, T.; Wegiel, J.; Hwang, Y. W. Harmine is an ATPCompetitive Inhibitor for Dual-Specificity Tyrosine Phosphorylation-Regulated Kinase 1A [Dyrk1A]. Arch. Biochem. Biophys. 2011, 507(2), 212-218.

16. Heshmati, E.; Abdolmaleki, A.; Mozdarani, H.; Sarvestani, A. S. Effects of Halogen Substitution on Watson-Crick Base Pairing: a Possible Mechanism for Radiosensitivity. Bioorg. Med. Chem. Lett. 2009, 19(17), 5256-5260.

17. Göckler, N.; Jofre, G.; Papadopoulos, C.; Soppa, U.; Tejedor, F. J.; Becker, W. Harmine Specifically Inhibits Protein Kinase DYRK1A and Interferes with Neurite Formation. FEBS J. 2009, 276(21), 6324-6237.

18. Jursic, B. S. Ab initio and Density Functional Theory Studies of the Potential Energy Sarface of the Linc $\rightarrow$ Linc Isomerization. J. Mol. Struct. 1998, 428, 42.

19. Jursic, B. S. Computing Transitoin State Structures whit Density Functionl Theory Methods. Theoretical and Computational Chemsitry 1996, 4, 709.

20. Jursic, B. S. Density Functional Theory and Complete Basis Set Ab initio Evaluation of Proton Affinity for Some Selected Chemical System. J. Mol. Struct. 1999, 487, 194.

21. Kurita, N.; Ivanov, P. M. Correlated Ab initio Molecular Orbital (MP3, MP4) and Density Functional (PW91, MPW91) Studies on the Conformations. J. Mol. Struct. 2000, 554, 184.

22. Ozimiski, W.; Garnuszek, P.; Bednarek, E.; Dobrowol, C. S. J. The Platinum Complexes whit Histamin: Pt(II) (Iodo-Hist) $\mathrm{cl}_{2}$ and Pt(IV) (Hist) $)_{2} \mathrm{cl}_{2}$ Inorg. Chim. Acta 2007, 360, 1904.

23. Becke, A. D. J. Chem. Phys. 1993, 9X, 5648.

24. Becke, A. D. Phys. Rev. A. 1988, 38, 3098.

25. Hohenberg, P.; Kohn, W. Phys. Rev. 1996, 136, B864; Kohn, W. and Sham, L. J. Phys. Rev. 1965, 140. All33; Stater, J. C. Quantum Theory of Molecules and Solids. The SelfConsistent Field for Molecular and Solids, Vol. 4; McGrawHill: New York, 1974.

26. Kurita, N.; Ivanov, P. M. Correlated Ab initio Molecular Orbital (MP3, MP4) and Density Functional (PW91, MPW91) Studies on the Conformations. J. Mol. Struct. 2000, 554, 184.

27. Perdew, J. P. Phys. Rev. B 1986, 33, 8822.

28. Perdew, J. P.; Wang, Y. Phys. Rev. B 1992, 45, 13244.

29. Lee, C.; Yang, W.; Parr, R. G. Phys. Rev. B. 1988, 37, 785; Miehlich, B.; Savin, A.; Sol1, H.; Preuss, H. Chem. Phys. Lett. 1989, 157, 200.

30. Perdew, J. P.; Zunger, A. Phys. Rev. B. 19XI, 23, 5048.

31. Vosko, S. H.; Wilk, L.; Nusair, M. Can. J. Phys. 1980, 58, 1200.

32. Nedelec, J. M.; Hench, L. L. Effect of Basise Set and of Electronic Correlation on Silica Rings. Journal of NonCrystaline Solids 2000, 277, 106. 